\title{
A NOVEL TECHNIQUE OF PROSTHETIC REHABILITATION OF A PATIENT WITH MICROSTOMIA USING SECTIONAL STOCK AND MODIFIED CUSTOM TRAY
}

\author{
DR. NITIKA GUPTA ${ }^{1}$, DR. HARIT TALWAR ${ }^{2} \&$ DR. NITASHA GANDHI ${ }^{3}$
}

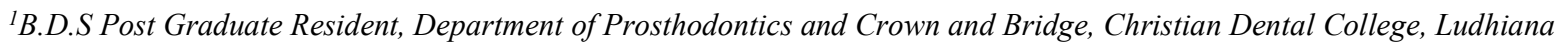
2B.D.S., M.D.S., Assistant Professor, Department of Prosthodontics and Crown and Bridge, Christian Dental College, Ludhiana

${ }^{3}$ B.D.S., M.D.S., Professor and Head, Department of Prosthodontics and Crown and Bridge, Christian Dental College, Ludhiana

\section{ABSTRACT}

A patient with microstomia is a challenge for the prosthodontist at every stage of rehabilitation. A contemporary technique using sectional stock tray loaded with putty material and a rigid sectional custom tray incorporated with press button assembly for extra oral stabilisation has been described. The above two techniques were used to impart ease of tray insertion and comfort to the patient during impression making procedures. Also, a prefabricated easily available hinge was incorporated into the denture bases to facilitate easy intra-oral placement of the prosthesis.

SUBHEADING: novel technique of microstomia management

KEYWORDS: microstomia, denture design, prosthodontics

Received: Jan 02, 2021; Accepted: Jan 21, 2021; Published: Feb 16, 2021; Paper Id.: IJDRDJUN20211

\section{INTRODUCTION}

A microstomia patient is no less than a challenge to a dental practitioner as it necessitates the need for modifications in conventional prosthodontic therapy. By definition microstomia is an abnormally small orifice which can be a resultant of various etiological factors such as orofacial neoplasms, cleft lip, maxillofacial trauma, burns, radiotherapy and scleroderma ${ }^{1}$. The perplexity which a microstomia patient poses for a dentist ranges from impression making till the insertion of denture bases ${ }^{2}$. Even though surgical intervention remains the most reliable method of long term management of a patient with microstomia, however, only a limited number of patients can afford the economic expense of undergoing surgical therapy. Hence, conservative management in the form of splinting and modified prosthodontic therapy has been advocated. Various tray designs and techniques of preliminary and final impression making procedures have already been suggested in literature ${ }^{2}$. Sectional, collapsible and swing-lock dentures are a few of the methods which have been proposed for the prosthetic rehabilitation of patients with microstomia ${ }^{3,4,5}$. However, there is not enough literature outlining the techniques for primary impression taking that are less time consuming, economical and easy to execute. This article describes the use of a novel technique of making preliminary impression with the combined use of a sectional stock tray with putty material, since an impression tray is the largest item requiring intra oral placement. Along with this, a method of custom tray fabrication using press button assembly for antero-posterior stabilisation has been described. This 
article also illustrates use of an easily available hinge for the fabrication of collapsible denture.

\section{CASE HISTORY:}

A 72 year old male patient with restricted mouth opening reported to the Department of Prosthodontics of Christian Dental College, Ludhiana for prosthetic rehabilitation. The patient gave history of squamous cell carcinoma of right buccal mucosa which was previously managed by radiotherapy and surgical resectioning.

The findings of the clinical examination suggested that the mouth opening was limited to $20 \mathrm{~mm}$ of inter-labial distance (Fig. 1), which was a problem for conventional prosthodontics therapy. The patient was completely edentulous with fibrous bands in the right buccal mucosa. The patient was not willing for conservative or surgical treatment, hence fabrication of collapsible denture with an altered impression technique was planned.

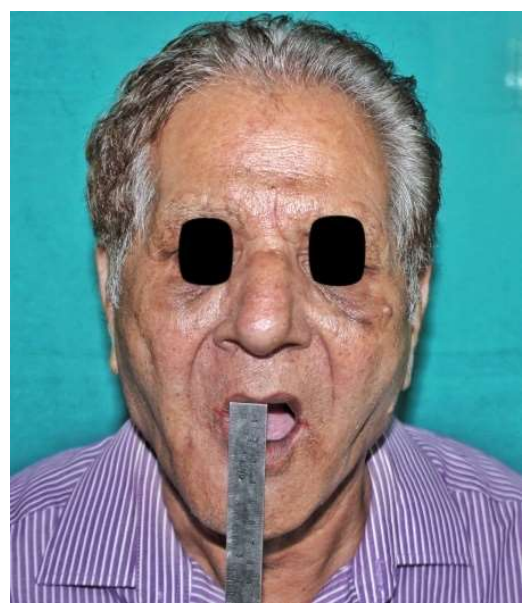

Figure 1: Pre-op photograph of the patient showing reduced mouth opening

\section{PROCEDURE}

1. For the primary impression, a stock sectional tray loaded with sufficient quantity of putty material (GC-Flexseed Putty Type Vinyl Polysiloxane Impression Material) was used, such that it covered all the important anatomic areas. The material loaded into the tray was placed in the patient's mouth and adapted using fingers over the area unsupported by the sectional tray and after polymerisation, it was loaded with injectable light body addition silicone(GC-Flexseed Light Body Vinyl Polysiloxane Impression Material) for recording finer details (Fig. 2). 
2.

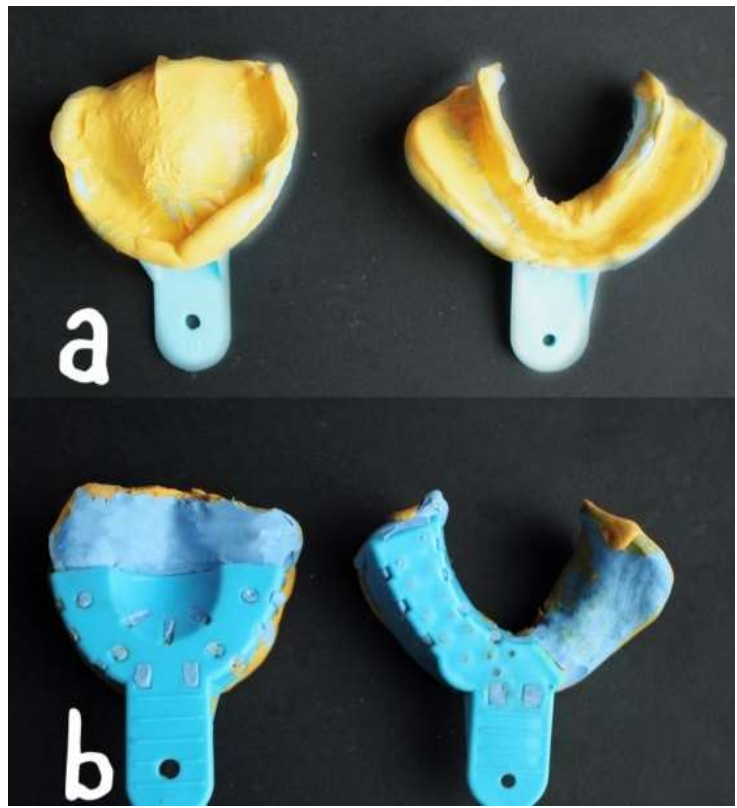

Figure 2: a) Front view of flexible preliminary impression made using sectional stock tray and polysiloxane impression material b) Back view of flexible preliminary impression made using sectional stock tray and polysiloxane impression material

3. The mandibular impression was recorded in a similar manner, with the sectional tray placed on one side, loaded with putty impression material on continuation with the other side (Fig. 2). The impressions were stabilised in a mix of dental stone type III ((Kalstone Dental Gypsum Type III, Kalabhai Karson, India) and then poured in dental plaster type II (DENTEX Dental Plaster Class II).

4. Sectional custom trays for both upper and lower arches were fabricated with autopolymerising resin ((DPI-RR Cold Cure, The Bombay Burmah Trading Corporation Ltd., Mumbai, Maharashtra, India) using the conventional sprinkle on method ${ }^{6}$. Press buttons (Snap Fasteners, K.S. Exports, Delhi, India) assembly were fixed on to the custom tray on the cuspid and first molar region using autopolymerising resin (Fig. 3a \& b).

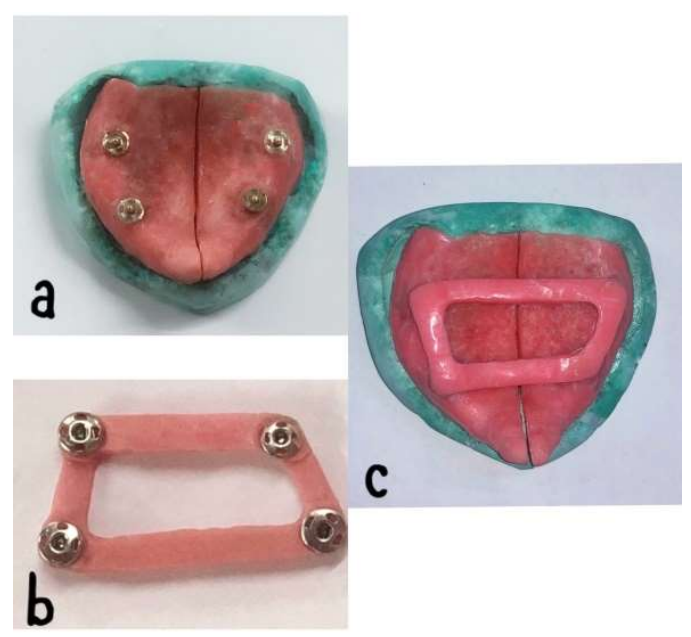

Figure 3: a) Sectional tray showing male component of press button assembly b) Trapezoidal acrylic jig incorporating female component c) Sectional tray assembled together using press button assembly 
6. The female component of the press button assembly was attached to an acrylic resin block fabricated using autopolymersing acrylic resin. The block which was trapezoidal in shape was fabricated to provide rigid stabilisation and a snap fit. Both the maxillary and mandibular sectional custom trays were then cut in half at the centre using diamond disk (Fig. 3 c).

7. Border moulding was carried out using conventional low fusing impression compound (DPI Pinnacle, The Bombay Burmah Trading Corporation Ltd., Mumbai, Maharashtra, India) with left and right sections placed consecutively. The wash impressions were recorded using metallic oxide paste (DPI impression paste, Dental Products of India Ltd.), by placing each half separately (Fig. 4).

8.

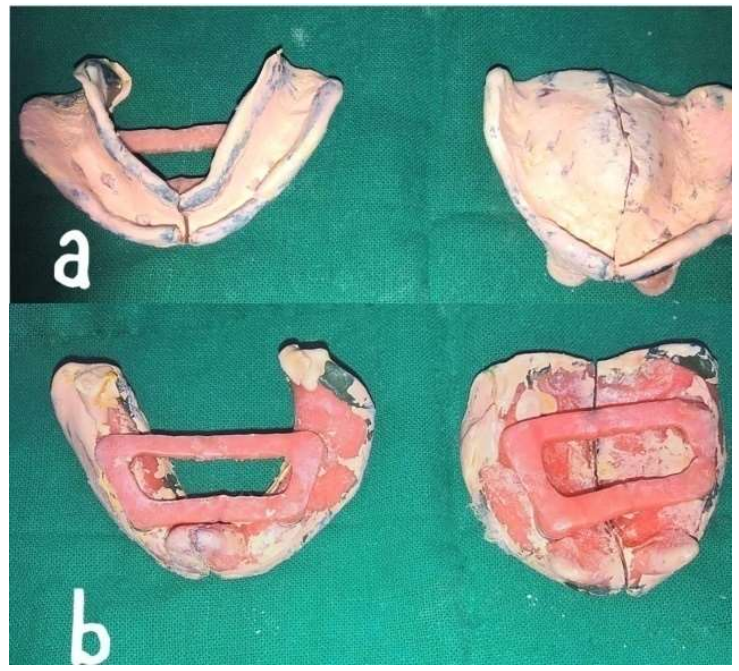

Figure 4: a) Border moulding and wash impression made in two sections b) and assembled extra orally using acrylic jig and press button assembly

9. After the impression paste was set, the two halves of the tray were assembled extra-orally using acrylic trapezoid shaped jig incorporated with female component of the press buttons (Fig. 4). The fit was verified with a snap sound of the press button assembly.

10. The maxilla-mandibular jaw relations were recorded using the conventional methods and no loss of vertical dimension of occlusion was noticed.

11. Trial denture bases were evaluated for proper occlusion, fit, aesthetics and phonetics.

12. The dentures were processed in a single piece using heat polymerised acrylic resin (DPI Heat Cure, Dental Products of India, Ltd.) using the conventional compression moulding technique. The patient could insert the mandibular denture as a single piece with some difficulty, as it could be rotated $90^{\circ}$ in a sagittal plane and then stabilised intra-orally. The maxillary denture could not be inserted as one piece and hence, a sectional denture was fabricated using a prefabricated hinge used in jewellery boxes (Fig. 5a).

13. The maxillary denture was stabilised on the master cast and a window was created in the mid-palatal region approximating the size of the hinge, which was incorporated into the denture using autopolymerising resin. 
14.

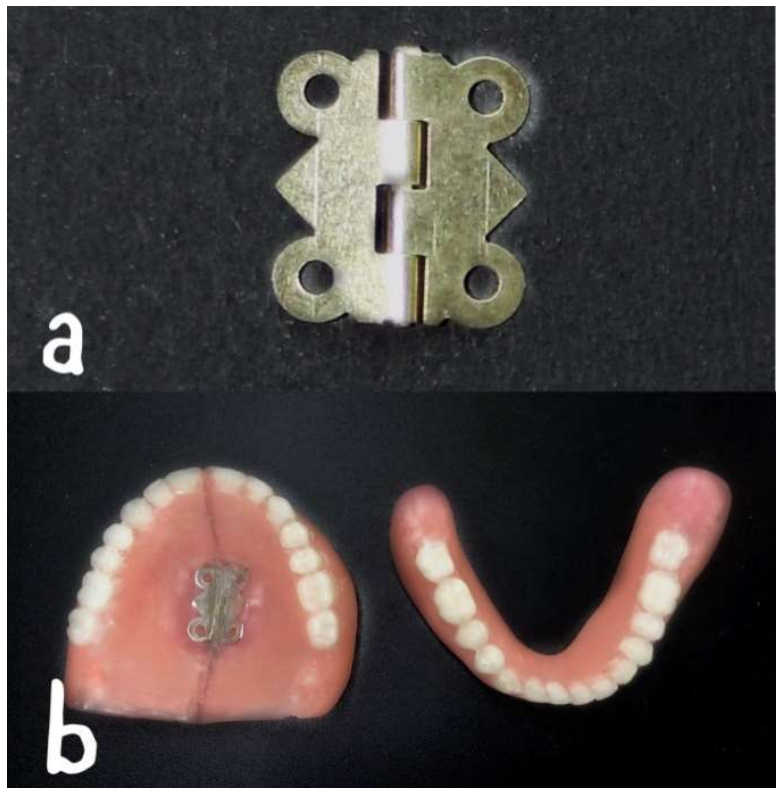

Figure 5: a) pre-fabricated hinge used in jewellery boxes b) maxillary collapsible denture and conventional mandibular denture

15. Following this, the maxillary denture was sectioned in half using a diamond disk (Fig. 5b). The finished and polished dentures were tried in the mouth for retention, stability and ease of insertion. (Fig. 6)

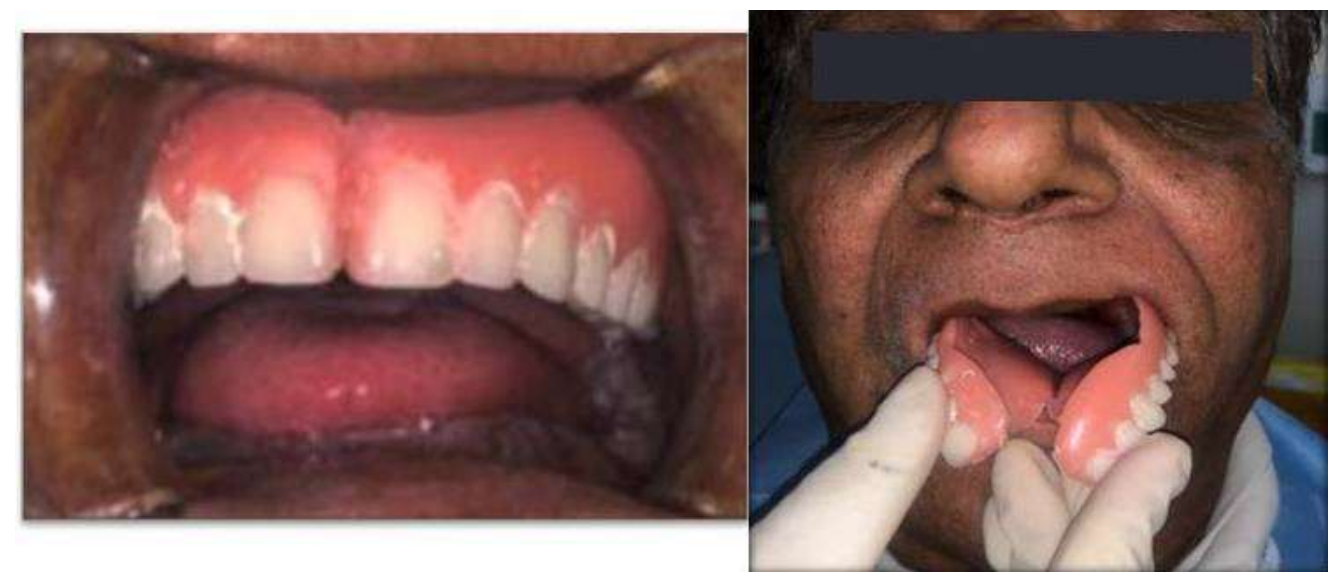

Figure 6: Intra-oral placement of definitive restoration easy with the use of collapsible denture

\section{DISCUSSIONS}

Rehabilitation for a microstomia patient, not undergoing surgical intervention is limited to modifications in the conventional prosthodontic therapy. Intraoral placement of a loaded impression tray is the first and the foremost problem faced by a dentist for a patient with reduced mouth opening $(<30 \mathrm{~mm})$. There is a lack of literature suggesting the methods of preliminary impression making in such patients. This article describes a semi-rigid tray, which is a combination of sectional stock tray and heavy viscosity putty material used for making preliminary impressions of the patient. Modification of stock trays by sectioning it into halves was not required in this study. Putty material has been used for taking preliminary impressions because it offers the advantage of adequate manipulation time and elasticity for ease of insertion into the oral cavity. The rigidity for facilitating the pouring of the impressions was provided by the stock sectional 
tray and the dental stone $\mathrm{mix}^{8}$.

A custom tray sectioned into two halves and assembled using press buttons was used for making wash impressions. In a study by Shreya S. Colvenkar ${ }^{9}$, the press buttons were incorporated only in the handle of the custom tray which could cause instability in the posterior section of the tray. The present study states that the press buttons should be incorporated in cuspid and molar regions to provide antero-posterior stabilisation on extra-oral assembly of the two sections of the custom tray.

Several sectional and collapsible denture designs have been presented in the literature incorporating clasps, rods, cast-locking recesses, using lock and pin attachments ${ }^{3,4,5,10}$. But their fabrication entails a complex, tedious and expensive process. This study illustrates the use of a pre-fabricated metal hinge used in commonly available jewellery boxes for the fabrication of a collapsible denture. The press buttons and pre-fabricated hinge used in this study are economical, easily available and simplified the laboratory procedures required for denture fabrication.

\section{CONCLUSIONS}

Prosthetic rehabilitation of a microstomia patient is a challenging task which can be easily managed by creative modifications in the conventional treatment. Use of a semi rigid sectional stock tray with putty material offers ease of impression making with commonly available materials in a dental clinic. Sectional custom tray with antero-posterior press button assembly can offer greater extra oral stabilisation and the incorporation of pre fabricated hinge to make a collapsible denture are viable and cost-effective alternatives.

\section{REFERENCES}

1. Academy of Prosthodontics. The glossary of prosthodontics terms. 7th ed. J Prosthet Dent 1999;81:39-110

2. Satish Kumar Nadimpalli, "Sanskrit: A Vaaradhi to English Vocabulary “, International Journal of Linguistics and Literature (IJLL), Vol. 5, Issue 4,pp. 33-38

3. Prithviraj DR, Ramaswamy S, Romesh S. Prosthetic rehabilitation of patients with microstomia. Indian Journal of Dental Research. 2009 Oct 1;20(4):483.

4. Kumar KA, Bhat V, Nair KC, Suresh R. Preliminary impression techniques for microstomia patients. The Journal of the Indian Prosthodontic Society. 2016 Jul;16(3):229.

5. Basavanna JM, Raikhy A. Sectional Denture for Microstomia Patient: A Clinical Report. International Journal of Prosthodontics \& Restorative Dentistry. 2013 Apr 1;3(2):62.

6. Satpathy A, Gujjari AK. Complete denture in a microstomia patient. Journal of Clinical and Diagnostic Research: JCDR. 2015 May;9(5):ZD16

7. Sharma A, Arora P, Wazir SS. Hinged and sectional complete dentures for restricted mouth opening: A case report and review. Contemporary clinical dentistry. 2013 Jan;4(1):74.

8. Zarb GA, Bolender CL, Eckert S, Jacob R, Fenton A, Stern RM. Prosthodontic

9. $\quad$ treatment for edentolous patient 12th ed. St. Louis: Mosby. 2003:202-3.

10. Whitsitt JA, Battle LW. Technique for making flexible impression trays for the microstomic patient. The Journal of prosthetic dentistry. 1984 Oct 1;52(4):608-9. 
11. Kotresh Sardar \& Rajashekar Patil, "Digital Manufacturing of Dental Implants using Computer Aided Engineering and Fused Deposition Method “, International Journal of Mechanical and Production Engineering Research and Development (IJMPERD), Vol. 8, Issue 4, pp. 905-912

12. Jawad K. Oleiwi, Qahtan Adnan Hamad \& Hadil Jabbar Abdul Rahman , "TENSILE PROPERTIES AND MORPHOLOGICAL TEST OF HEAT CURED ACRYLIC RESIN REINFORCED BY NATURAL POWDERS “, International Journal of Mechanical and Production Engineering Research and Development (IJMPERD), Vol. 8, Issue 6, pp.325-334

13. Colvenkar SS. Sectional impression tray and sectional denture for a microstomia patient. Journal of Prosthodontics: Implant, Esthetic and Reconstructive Dentistry. 2010 Feb;19(2):161-5

14. Tulunoglu I, Lee MH, Taifur ML, Tulunoglu O. Restoring masticatory function in a patient with severe microstomia using rapid prototyped mesh and a custom-made hinge and swing-lock prosthesis. The Journal of prosthetic dentistry. 2018 Jun $1 ; 119(6): 887-92$. 
